\title{
The Effect of Wound Dressings on a Bio-Engineered Human Dermo-Epidermal Skin Substitute in a Rat Model
}

\author{
Martina Hüging, MD, * $\dagger$ Thomas Biedermann, PhD, $\ddagger$ Monia Sobrio, MSc, $† \ddagger$ \\ Sarah Meyer, BSc, $\ddagger$ Sophie Böttcher-Haberzeth, MD,${ }^{*} \dagger \ddagger$ Edith Manuel, ${ }^{*} \dagger$ \\ Maya Horst, MD, $\uparrow \$$ Sally Hynes, $M D,{ }^{*} \dagger$ Ernst Reichmann, PhD, $†$ \\ Clemens Schiestl, MD, ${ }^{\star} †$ Fabienne Hartmann-Fritsch, PhD†‡
}

\begin{abstract}
Autologous bio-engineered dermo-epidermal skin substitutes are a promising treatment for large skin defects such as burns. For their successful clinical application, the graft dressing must protect and support the keratinocyte layer and, in many cases, possess antimicrobial properties. However, silver in many antimicrobial dressings may inhibit keratinocyte growth and differentiation. The purpose of our study was to evaluate the effect of various wound dressings on the healing of a human hydrogel-based dermoepidermal skin substitute in preparation for the first-in-human clinical trials. Human dermo-epidermal skin substitutes approved for clinical trials were produced under good manufacturing practice conditions, transplanted onto immuno-incompetent rats, and dressed with either Vaseline Gauze $^{\mathrm{TM}}$ (Kendall Medtronic, Minneapolis, USA), Suprathel ${ }^{\circledR}$ (PolyMedics Innovations GmbH, Denkendorf, Germany), Urgotul ${ }^{\circledR}$ SSD (Urgo Medical, Shepshed, United Kingdom), Mepilex ${ }^{\circledR}$ AG (Mölnlycke Health Care, Gothenburg, Sweden), or Acticoat ${ }^{\mathrm{TM}}$ (Smith\&Nephew, Baar, Switzerland). Grafts were assessed clinically for take, epithelialization, and infection at 10 and 21 days post-transplantation, and histologically at 21 days. There were three subjects each in the Vaseline Gauze ${ }^{\mathrm{TM}}$ and Suprathel ${ }^{\circledR}$ groups, and four subjects each in the Urgotul ${ }^{\circledR}$ SSD, Mepilex ${ }^{\circledR}$ AG, and Acticoat ${ }^{\mathrm{TM}}$ groups. For all samples, the take rate was $100 \%$ and the expected keratinocyte number, epithelialization and epidermal stratification were observed. All of the dressings in the current study were well tolerated by our human dermo-epidermal skin substitute. The tolerance of the silver-based dressings is particularly relevant given the high risk of bacterial contamination with large skin defects, and provides pivotal information as we embark on clinical trials for this novel skin substitute. (J Burn Care Res 2017;38:354-364)
\end{abstract}

The rapid and permanent coverage of large fullthickness skin defects, such as those resulting from burns, scar revisions, avulsion injuries, or the excision

\footnotetext{
From the *Pediatric Burn Center, Division of Plastic and Reconstructive Surgery, Department of Surgery, University Children's Hospital Zurich, Switzerland; +Children's Research Center, University Children's Hospital Zurich, Switzerland; $\neq$ Tissue Biology Research Unit, Department of Surgery, University Children's Hospital Zurich, Switzerland; and \$Department of Surgery, University Children's Hospital Zurich, Switzerland. This study was financially supported by the EU-FP7 project EuroSkinGraft (FP7/2007-2013: grant agreement no. 279024), and by the Clinical Research Priority Programs (CRPP) of the University of Zurich, Switzerland.

Hüging and Biedermann contributed equally to this study.

Address correspondence to Fabienne Hartmann-Fritsch, Tissue Biology Research Unit, Department of Surgery, University Children's Hospital Zurich, August Forel-Strasse 7, 8008 Zurich, Switzerland. Email: fabienne.hartmann@kispi.uzh.ch.

Copyright (C) 2017 by the American Burn Association 1559-047X/2017
}

DOI: $10.1097 / B C R .0000000000000530$ of skin tumors, is a major challenge in burn, plastic and reconstructive surgery. The use of autologous split-thickness skin grafts is currently the standard method for coverage of large defects. However, in severe burn injuries, in particular, the shortage of donor sites can be a substantial limitation of this technique.

The clinical introduction of cultured epithelial autografts (CEAs) in the 1980s helped address donor site limitations and improve survival in patients with severe burns. ${ }^{1-3}$ However, more than 30 years later, despite tremendous research efforts, CEA remains fraught with considerable problems when used independently for the coverage of deep burns, including graft fragility, poor take, susceptibility to infection, instability of the healed grafts, and, ultimately, unsatisfactory longterm functional and aesthetic results. ${ }^{4-6}$ The development of autologous bio-engineered dermo-epidermal 
skin substitutes has provided a promising alternative to CEAs to additionally improve the aesthetic and functional outcomes in burn treatment.

Boyce et $\mathrm{al}^{4-6}$ added patient's own fibroblasts and keratinocytes on a collagen-glycosaminoglycan substrate to produce and clinically test autologous dermo-epidermal skin substitutes. Although promising clinical trials, in terms of graft take and scar appearance, have been conducted, these skin substitutes are still not available for commercial use.

Apligraf, a dermo-epidermal skin substitute, is U.S. Food and Drug Administration (FDA) approved and commercially available for broader clinical application. ${ }^{7-10}$ However, it is only a transient coverage, as it does not consist of patient's own cells, but allogenic human cells.

Therefore, 15 years of laboratory research have been invested in our institute in Zurich in the engineering of a bilayered skin substitute. Our research team has succeeded in growing a large hydrogelbased human dermo-epidermal skin construct featuring a multilayered stratified epidermis and a near-normal dermis. These grafts have been successfully transplanted onto immuno-incompetent rats in several preclinical animal studies. ${ }^{11-15}$

Currently, no comparable, autologous bio-engineered dermo-epidermal skin substitute is commercially available for clinical application. For this reason, we cannot rely on former experience regarding clinical handling. ${ }^{16}$ During the development of the new skin substitute in the animal model, grafts were enclosed by a small steel ring, which was sutured on the back of the rats for protection, and covered with simple silicon foil. However, these measures are not suitable for clinical use.

Since the newly developed dermo-epidermal skin substitute contains cultured autologous epithelial cells in addition to fibroblasts, the dressing requirements for the clinical application will be at least as rigorous as for CEAs. CEAs are highly sensitive to tangential forces and infection, both of which are directly correlated with poor graft take. Therefore, the requirements of a CEA wound dressing are much more stringent than those of a split-thickness skin graft dressing. ${ }^{17,18}$ Protection against sheering forces and provision of an optimal wound healing microenvironment are critical to the healing of CEAs. In addition, the dressing should exhibit antibacterial properties, given the vulnerability of CEAs to bacterial contamination and the associated cytotoxins and proteases. ${ }^{19-23}$ The use of antimicrobial silver-containing dressings is routine in burn treatment. ${ }^{24-26}$ However, studies performed to determine whether silver is harmful to keratinocytes have revealed mixed results. ${ }^{27-31}$
In summary, the ideal dressing for bio-engineered skin combines various characteristics, including protection against sheering forces, provision of an optimal wound healing microenvironment, antibacterial properties, and minimal cytotoxicity. The purpose of the current study is to evaluate which dressing best unites the aforementioned properties, in preparation for the first clinical application of this novel skin analog. In particular, we aim to determine the potential cytotoxic effect of dressings containing silver on our bio-engineered skin construct.

\section{METHODS}

\section{Cell Isolation and Culture}

Human split-thickness skin samples were harvested after informed consent from patients and/or legal guardians was obtained. Human primary fibroblasts and keratinocytes were isolated as previously described. ${ }^{11}$ Isolation and cell culture was performed in a clean room laboratory class $\mathrm{A}$, in line with good manufacturing practice (GMP) guidelines, ${ }^{32}$ according to the defined standard operating procedures and in compliance with the established quality management system. Cell cultures were performed from four independent donor samples.

\section{Dermo-Epidermal Skin Substitutes}

Human dermo-epidermal skin substitutes comprised of human keratinocytes seeded onto collagen type I hydrogels with incorporated human dermal fibroblasts were created as previously described. ${ }^{33}$ The skin substitutes were engineered in a laboratory class A clean room in line with GMP guidelines, according to the defined standard operating procedures and in compliance with the established quality management system (GMP facility of the Wyss Translational Center Zurich-Regenerative Medicine Technologies Platform, University of Zurich, Zurich, Switzerland). Briefly, $10^{6}$ human primary dermal fibroblasts (passage 2-3) were mixed with bovine collagen type I (Symatese, Chaponost, France) and neutralized with a buffer containing $\mathrm{NaOH}$. The solution was poured into a cell culture insert $(7 \times 8 \mathrm{~cm})$ with a pore size of $3 \mu \mathrm{m}$ (Oxyphen, Wetzikon, Switzerland). After incubation for 15 minutes at $37^{\circ} \mathrm{C}$ with $5 \% \mathrm{CO}_{2}$, the hydrogels were plastically compressed. ${ }^{12}$ The hydrogels were cultivated in Dulbecco Modified Eagle Medium (DMEM) supplemented with 10\% Fetal Calf Serum (FCS) (Invitrogen, Basel, Switzerland). Media changes were performed every 2 to 3 days. Five days thereafter, $8 \times 10^{6}$ human primary keratinocytes (passage $2-3$ ) were seeded onto the complete surface of 
the hydrogels. Hydrogels were cultivated for 5 days with DMEM + 10\% FCS in the lower chamber and keratinocyte medium (Cell'n'Tec, Bern, Switzerland) in the upper chamber, before transplantation onto immuno-incompetent rats. For transplantation, round grafts of approximately $24 \mathrm{~mm}$ diameter were cut from the dermo-epidermal skin substitutes.

\section{Transplantation}

All animal experiments were approved by the local Committee for Experimental Animal Research. The preparation of immuno-incompetent $\mathrm{nu} / \mathrm{nu}$ rats (age 8-10 weeks, Harlan) was performed as previously described. ${ }^{34}$ A surgical skin incision on the back of the rat was performed, the panniculus carnosus was kept intact. Steel rings with a diameter of $26 \mathrm{~mm}$ were implanted into full-thickness skin defects on the back of the rats to prevent wound closure by ingrowth of rat skin into the wound area. Grafts were transplanted onto the panniculus carnosus into the metal rings, fixed with sutures and covered with one of the study wound dressings (Figure 1).

\section{Wound Dressings}

For coverage of the transplanted skin substitute, various wound dressings were used, as summarized in Table 1. The wound dressings were in direct contact with the epidermis of the skin substitute. Any remaining space in the chamber was filled with Jelonet $^{\text {TM }}$ (Smith \& Nephew, Baar, Switzerland). All used dressings were applied according to the instructions of use. All wound dressings were applied dry, except Acticoat $^{\mathrm{TM}}$, which was moistened with sterile Aqua dest before placement onto the graft. Square pieces of Urgotul ${ }^{\circledR}$ and Ligasano ${ }^{\circledR}$ were fixed onto the chamber to protect the graft and chamber. As positive control, skin substitutes were covered with silicon foil, which is the standard dressing in our model as previously published. ${ }^{11,13-15,33,35}$

The first dressing change was performed on day 10. Thereafter, the grafts were covered exclusively with silicon foil, since grafts are less susceptible to sheering forces and infection at this stage.

\section{Clinical Evaluation}

On days 10 and 21, wound dressing changes and photographic documentation were performed. The percent graft take and presence of local signs of infection were assessed at the first dressing change on day 10. At day 21, the grafts were assessed for percent epithelialization and infection. Animals were killed 21 days post-transplantation.

\section{Histology}

Grafts were excised, halved, and embedded in Optimum cutting temperature (OCT) compound (Tissue-Tek ${ }^{\circledR}$, Sakura Finetek, Japan) or fixed in 4\% paraformaldehyde (Medite Medizintechnik AG, Nunningen, Switzerland) and then embedded in paraffin (McCormick, Richmond, VA). Ten micrometer paraffin sections were cut and stained with hematoxylin and eosin (Sigma-Aldrich, Buchs, Switzerland).

For immunofluorescence staining, OCT embedded tissue was frozen at $-20^{\circ} \mathrm{C}$, and sectioned at $10 \mu \mathrm{m}$. Permeabilization was performed in ice-cold acetone for 5 minutes. Thereafter, sections were

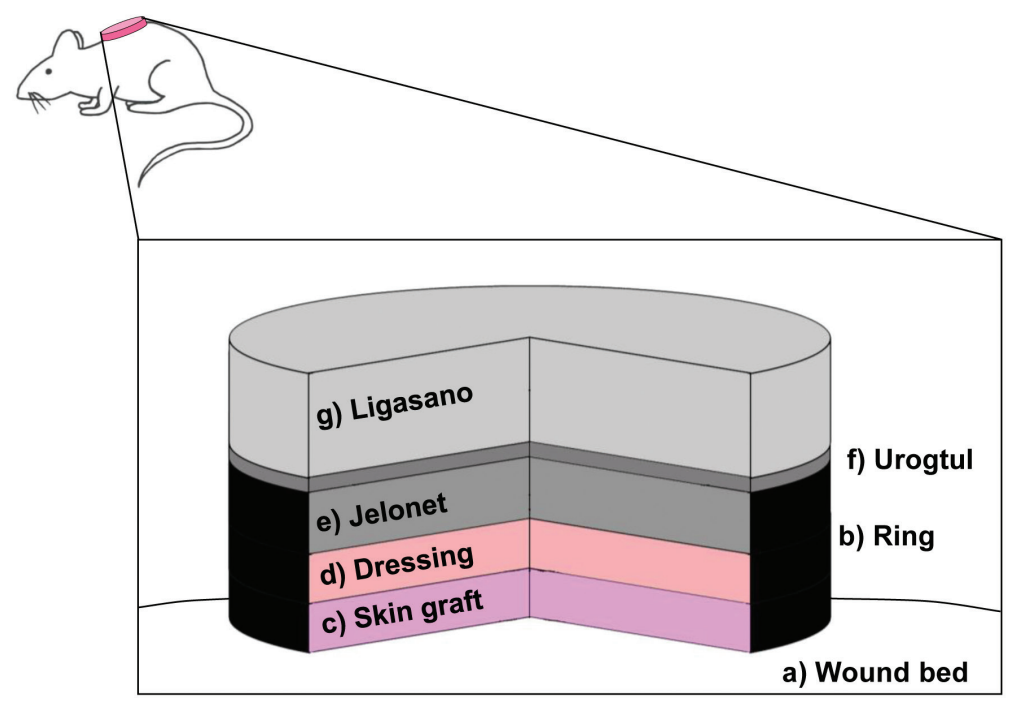

Figure 1. Schematic diagram of experimental set up. The full-thickness wound (a) is held open by a steel ring (b). The transplanted and sutured dermo-epidermal skin substitute (c) is covered with the wound dressing under investigation (d), followed by Jelonet ${ }^{\mathrm{TM}}$ (e) as needed to fill the chamber, and finally protected with a layer of Urgotul ${ }^{\circledR}(\mathrm{f})$ and Ligasano ${ }^{\circledR}(\mathrm{g})$. 
Table 1. Overview on used dressing materials

\begin{tabular}{|c|c|c|c|c|}
\hline Dressing & Manufacturer & Composition & Silver Content & $\begin{array}{l}\text { Number of } \\
\text { Animals }\end{array}$ \\
\hline \multicolumn{5}{|c|}{ Dressing material in direct contact with the skin substitute } \\
\hline Vaseline Gauze ${ }^{\mathrm{TM}}$ & Kendall & $\begin{array}{l}\text { Gauze impregnated with petrolatum }(8884413605 \text {, } \\
7.6 \times 22.9 \mathrm{~cm})\end{array}$ & - & 3 \\
\hline Suprathel ${ }^{\circledR}$ & $\begin{array}{l}\text { PolyMedics Innovations } \\
\text { GmbH }\end{array}$ & Lacto-capromer tetrapolymer & - & 3 \\
\hline Urgotul $^{\circledR}$ SSD & URGO Medical & $\begin{array}{l}\text { Polyester mesh impregnated with dispersion of } \\
\text { carboxymethyl cellulose, petroleum jelly, silver } \\
\text { sulfadiazine particles }\end{array}$ & $0.35 \mathrm{mg} / \mathrm{cm}^{2}$ & 4 \\
\hline Mepilex $^{\circledR}$ AG & Mölnlycke Health Care & $\begin{array}{l}\text { Polyurethane foam, polyethylene release film, silicone } \\
\text { gel, silver sulfate, activated charcoal }\end{array}$ & $1.2 \mathrm{mg} / \mathrm{cm}^{2}$ & 4 \\
\hline Acticoat ${ }^{\mathrm{TM}}$ & Smith \& Nephew & $\begin{array}{l}\text { Polyethylene nets coated with nanocrystalline silver, } \\
\text { absorbent inner core }\end{array}$ & $1.0-1.2 \mathrm{mg} / \mathrm{cm}^{2}$ & 4 \\
\hline \multicolumn{5}{|c|}{ Dressing material not in direct contact with the skin substitute } \\
\hline Jelonet $^{\mathrm{TM}}$ & Smith \& Nephew & $\begin{array}{l}\text { Low adherent, paraffin impregnated tulle gras dressing } \\
\text { made from open weave gauze }\end{array}$ & - & - \\
\hline Ligasano $^{\circledR}$ & $\begin{array}{l}\text { Ligamed medical Produkte } \\
\text { GmbH }\end{array}$ & Polyurethane foam & - & - \\
\hline Urgotul $^{\circledR}$ & URGO Medical & $\begin{array}{l}\text { Polyester mesh impregnated with dispersion of } \\
\text { carboxymethyl cellulose, petroleum jelly }\end{array}$ & - & - \\
\hline
\end{tabular}

air-dried and washed three times in phosphate buffered saline (PBS). After blocking with PBS containing $2 \%$ bovine serum albumin (Sigma) for 30 minutes at room temperature, sections were incubated with the prelabeled antibodies for 1 hour at room temperature. Sections were then washed three times with PBS, and nuclei were stained with $1 \mu \mathrm{g} / \mathrm{ml}$ Hoechst 33341 (Sigma) in PBS for 5 minutes at room temperature. Sections were then washed two times with PBS, and finally mounted with Dako fluorescent mounting solution (Dako, Baar, Switzerland).

\section{Antibodies}

The following antibodies were used: anti-human $\mathrm{Kl}$ (clone LHKl, 1:200, Novus Biologicals, Littleton, CO); anti-human Lam5a3 (clone P3H9-2, 1:100, Santa Cruz, Labforce AG, Nunningen, Switzerland); anti-rat CD31 (clone TDL-3A12, 1:50, BD Biosciences Pharmingen, Basel, Switzerland); anti-human CD90 (clone AS02, 1:50, Dianova, Hamburg, Germany); anti-human involucrin (clone SY5, 1:100, LabVision, Fremont, CA); anti-human loricrin (polyclonal, 1:500, Abcam, Cambridge); anti-rat CDllb (macrophage marker, 1:50, Santa Cruz, Labforce AG), and anti-rat HIS48 (granulocyte marker, 1:50, Santa Cruz, Labforce AG).

For immunofluorescence staining, primary antibodies were prelabeled with either Alexa488 or Alexa555 conjugated polyclonal goat $\mathrm{F}\left(\mathrm{ab}^{\prime}\right) 2$ fragments, according to the manufacturer's instructions (Zenon Mouse IgG Labelling Kit, Molecular Probes, Invitrogen, Basel, Switzerland).

\section{Microscopy}

The histochemical and immunofluorescence stainings were analyzed with a Nikon Eclipse TE2000-U inverted microscope, equipped with Hoechst, Fluorescein isothiocyanate (FITC) and Tetramethylrhodamine (TRICT) filter sets and connected with a DXMI200F digital camera (Nikon AG, Egg, Switzerland).

\section{RESULTS}

A total of 18 rats were studied. The breakdown by dressing group is shown in Table 1.

\section{Macroscopic Appearance of Epidermis on Transplanted Grafts}

Graft take of $100 \%$ with complete epithelialization was observed at 10 days for all dressing groups, including Vaseline Gauze ${ }^{\mathrm{TM}}$, Suprathel ${ }^{\circledR}$, Urgotul ${ }^{\circledR}$ SSD, Mepilex ${ }^{\circledR}$ AG, and Acticoat ${ }^{\mathrm{TM}}$ (Figure 2, second column). This is comparable to the positive control of grafts covered with silicon foil (data not shown), as previously published. ${ }^{11,13-15,33,35}$ No signs of local infection were observed. On grafts covered with Vaseline Gauze ${ }^{\mathrm{TM}}$, Urgotul ${ }^{\circledR}$ SSD, or Acticoat ${ }^{\mathrm{TM}}$, imprints of the mesh structure of the dressing were visible, whereas grafts covered with Mepilex ${ }^{\circledR}$ AG and Suprathel ${ }^{\circledR}$ did not show any imprint of the wound dressing.

At day 21, no signs for infection could be observed, and complete epithelialization was seen macroscopically for all groups (Figure 2, third column). Grafts 


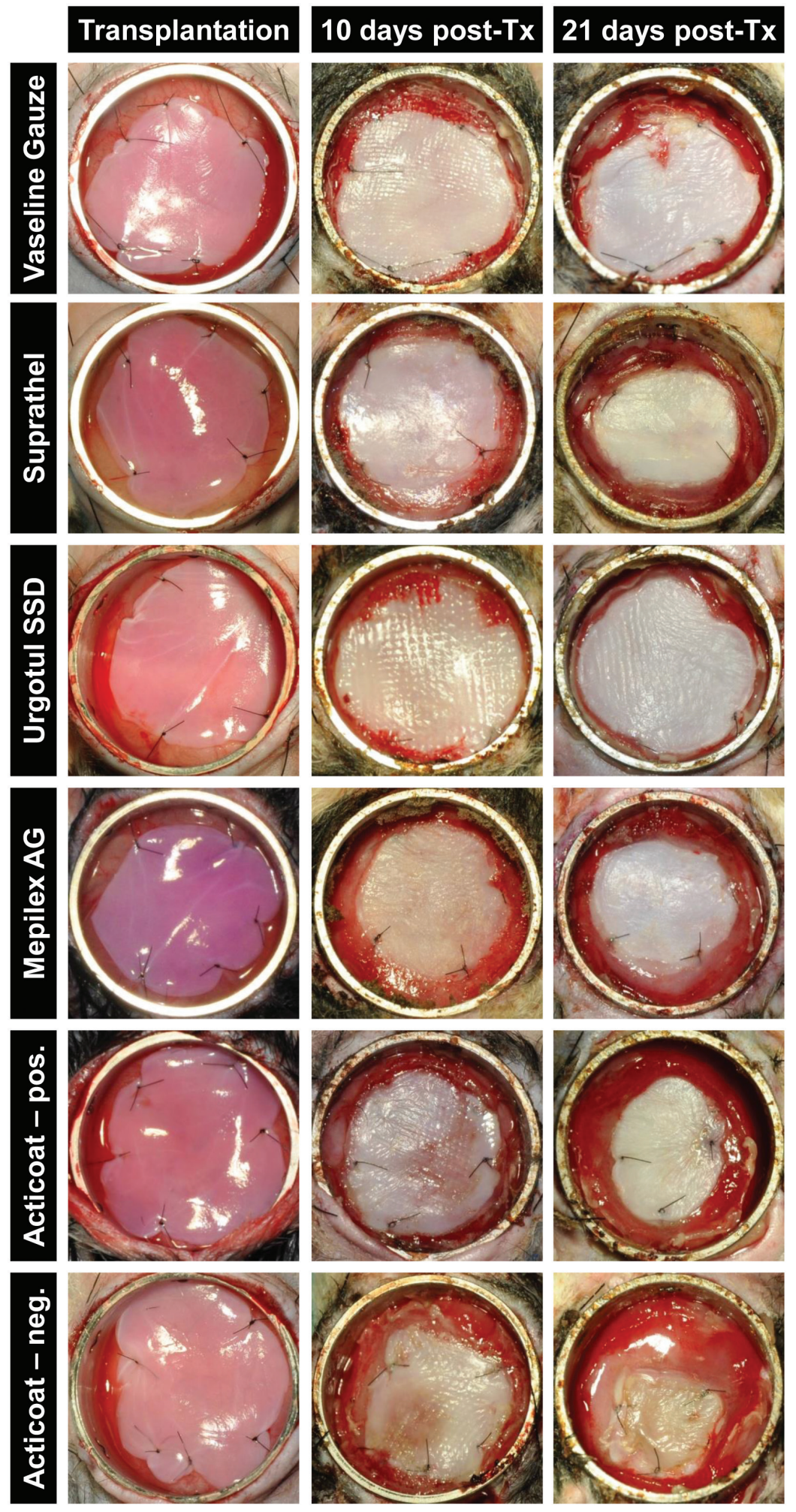

Figure 2. Macroscopic appearance of transplanted skin grafts. The macroscopic appearance of transplanted dermo-epidermal skin grafts at day of transplantation (left column), 10 days post-transplantation (middle column), and 21 days post-transplantation (right column) is shown. All transplants have a similar appearance on the day of transplantation. For transplants covered with Vaseline Gauze ${ }^{\mathrm{TM}}$, Suprathel ${ }^{\circledR}$, Urgotul ${ }^{\circledR}$ SSD, or Mepilex ${ }^{\circledR}$ AG, at 10 and 21 days post-transplantation the surface appears dry and dull. The surface of transplants covered with Acticoat ${ }^{\mathrm{TM}}$ appears to be less stable 10 and 21 days post-transplantation and inter-animal variations are higher (therefore representative apparent positive and apparent negative outcomes with Acticoat ${ }^{\mathrm{TM}}$ are shown). Diameter of metal ring is $26 \mathrm{~mm}$. 
covered with Acticoat ${ }^{\mathrm{TM}}$ showed diverse appearances macroscopically, in contrast to the more consistent appearance of grafts in the other groups (Figure 2, bottom two rows).

\section{Histological Analysis of Grafts Excised 21 Days Post-Transplantation}

Hematoxylin \& eosin stainings of cryosections revealed a multilayered, stratified epidermis, including a stratum corneum, for all samples (Figure 3 ). No significant differences could be identified between the various wound dressing groups. Interestingly, excised grafts covered with Acticoat ${ }^{\mathrm{TM}}$ also showed a well stratified epidermis, in spite of the variable macroscopic appearances (Figure 2).

\section{Immunofluorescence Analysis}

The immunofluorescence stains for the basal lamina component laminin 5 revealed a continuous deposition of a basement membrane in all grafts, independent of the wound dressing used (Figure 4, left column). Stains for keratin 1 showed expression of keratin $I$ in all suprabasal cell layers of the epidermis, indicating correct stratification in all groups (Figure 4, left column).

Expression of human-specific CD90 confirmed the presence of human dermal fibroblasts in all groups. Ingrowth of host blood vessels into the transplanted grafts, as detected by rat-specific CD31 staining, could be observed in all groups. Rat blood vessels in the human graft, in close proximity to the epidermis, were observed in samples from the Vaseline Gauze ${ }^{\mathrm{TM}_{-}}$, Suprathel ${ }^{{ }_{-}}$, Urgotul ${ }^{\circledR}$ SSD-, and Acticoat ${ }^{\mathrm{TM}}$-groups (Figure 4, right column). In samples from the Mepilex ${ }^{\circledR}$ AG-group, blood vessels were detected in the graft but not as close to the surface of the graft as was observed for the other groups.

In healthy human epidermis, involucrin is expressed in the epidermis from the last layer of the stratum spinosum to the first layers of the stratum granulosum. In all grafts, continuous involucrin expression was observed in the upper layers of the newly formed epidermis. Loricrin expression, a marker for cornified epithelial cells, was also similar in all grafts.

Granulocytes were stained with the granulocyte marker HIS48. Almost no granulocytes were found in the grafts. In samples covered with Acticoat ${ }^{\mathrm{TM}}$, some granulocytes could be detected (Figure 5, left column).

Host macrophages were stained with the macrophage marker integrin CDllb. No macrophages were detected in any of the grafts (Figure 5, right column). For all stainings, rat skin was used as a positive control (data not shown).
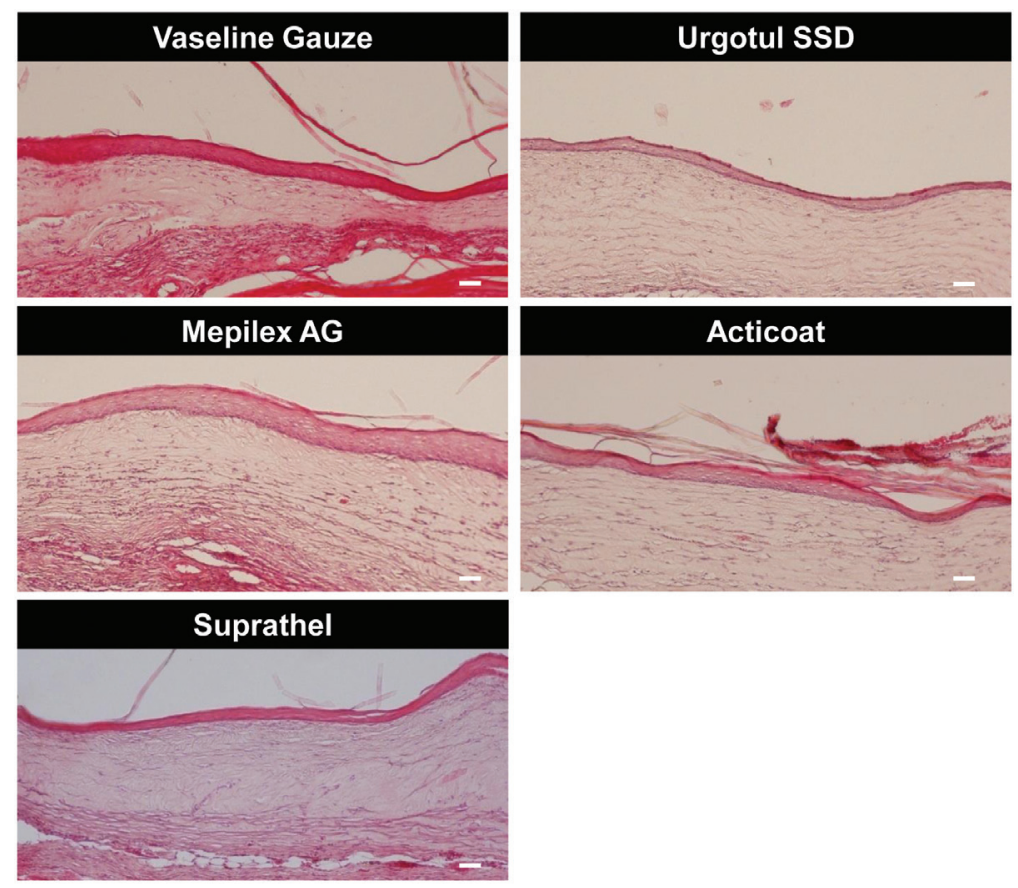

Figure 3. Histologic appearance of transplanted skin grafts. The histologic appearance of transplanted dermo-epidermal skin grafts 21 days post-transplantation is shown. Hematoxylin and eosin staining reveals a multilayered, stratified, and cornified epidermis for all samples. No differences can be observed regarding layers or status of stratification. Scale bars $50 \mu \mathrm{m}$. 

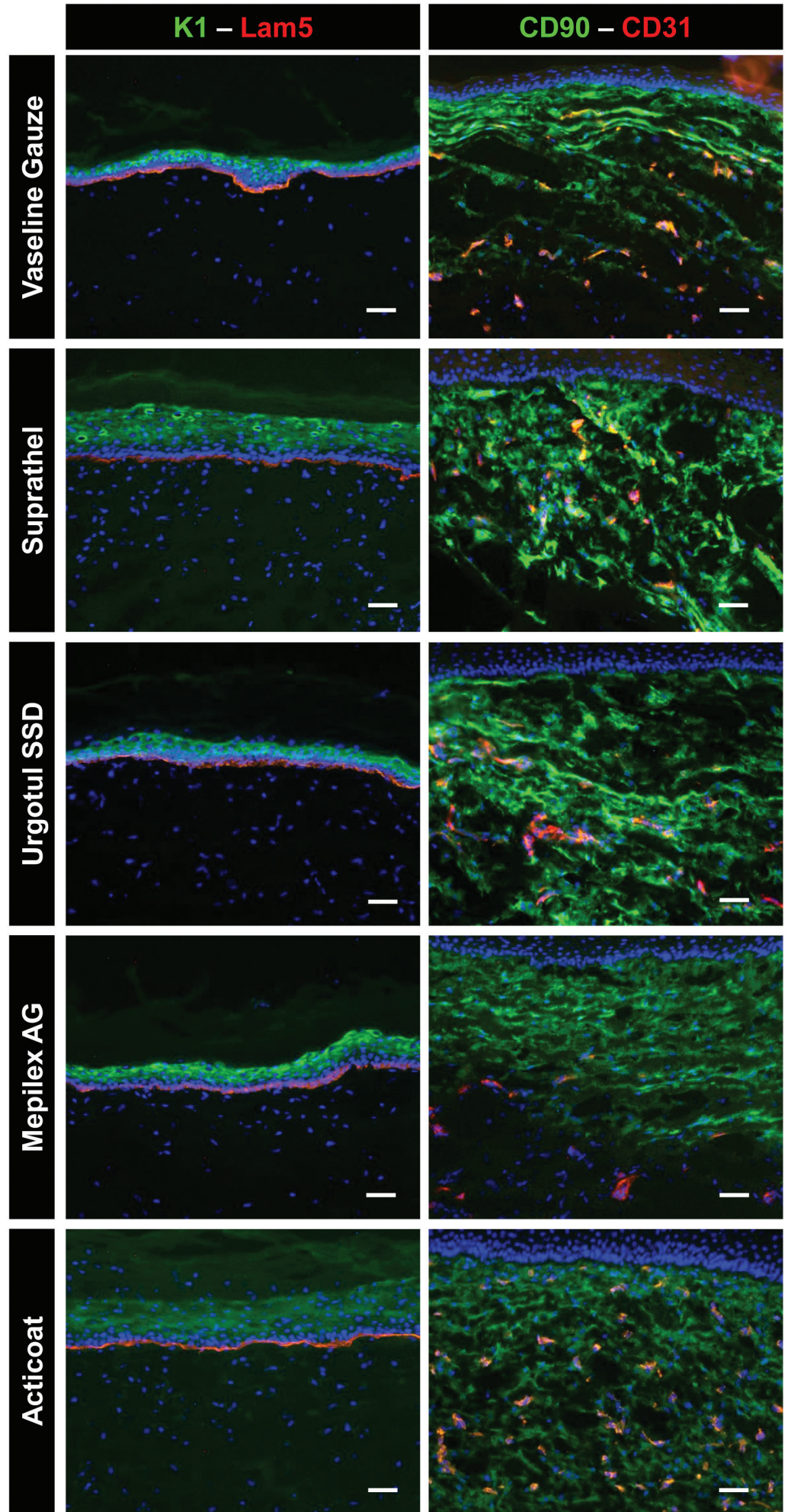

Figure 4. Immunofluorescence analysis of transplanted skin grafts. Immunofluorescence stainings of transplanted dermoepidermal skin grafts 21 days post-transplantation are shown. Keratin 1 expression (left column, green) was found in the suprabasal layers of all grafts. In all grafts, a continuous basement membrane was deposited as revealed by staining for the basement membrane component laminin 5 (left column, red). Remnants of the human graft could be detected by staining for human fibroblast-specific CD90 (right column, green) in all grafts. Rat blood vessels, as detected with rat-specific CD31 (right column, red), grew into the graft in all samples. Scale bars $50 \mu \mathrm{m}$. 


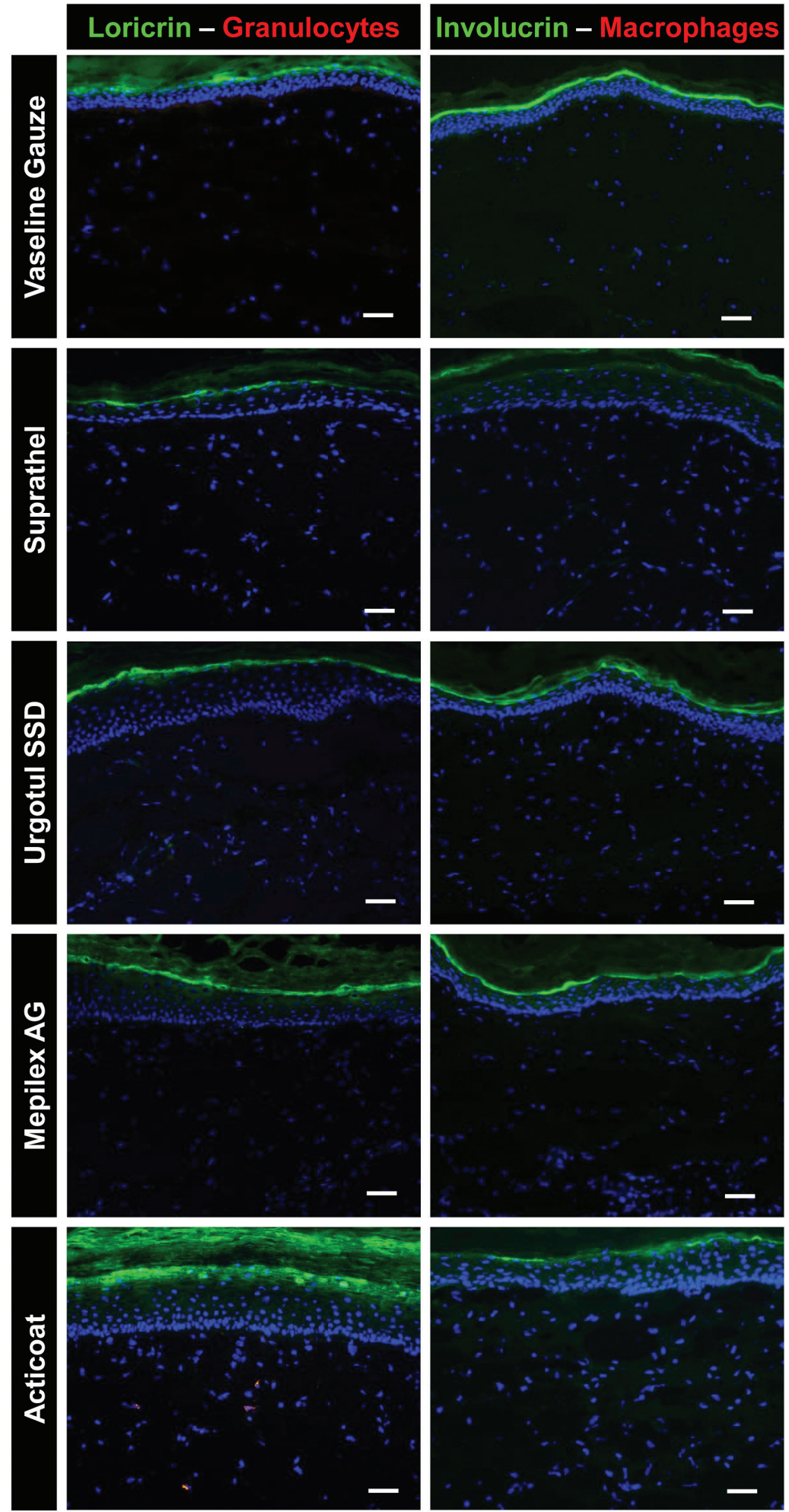

Figure 5. Immunofluorescence analysis for leukocytes in transplanted skin grafts. Immunofluorescence stainings of transplanted dermo-epidermal skin grafts 21 days post-transplantation are shown. Continuous loricrin expression was found in all grafts (left column, green). Granulocytes were stained with the granulocyte-specific marker HIS48. Only in Acticoat ${ }^{\mathrm{TM}}$ samples could some granulocytes be detected (left column, red). Involucrin expression was found in upper layers of the epidermis (right column, green). Macrophages were stained with the macrophage-specific marker CDIlb, but no macrophages were detected in the grafts (right column, red). Scale bars $50 \mu \mathrm{m}$. 


\section{DISCUSSION}

In several previous studies we demonstrated the use of our newly developed bio-engineered human dermo-epidermal skin substitute in preclinical in vivo assays. ${ }^{11,13-15,33}$ Our model offers the possibility to transplant human hydrogel-based dermoepidermal skin substitutes, which is unique and not comparable to any other model. Other authors have used plastic chambers transplanted onto mice, therefore the human skin substitutes were much smaller, ${ }^{36}$ or sutured human skin directly to the mice skin without any physical demarcation by ring or chamber, not preventing ingrowth of mice skin. ${ }^{37}$ We continuously modified and improved our skin constructs in order to obtain the best overall biological properties (eg, stability against sheering forces, promotion of vascular ingrowth). We were able to produce dermo-epidermal skin substitutes on a large scale by employing plastically compressed collagen hydrogels. ${ }^{12,38,39}$

The aim of this study was to determine the effect of several wound dressings, which are commonly used for skin graft coverage, on our dermo-epidermal skin constructs in our in vivo transplantation model. The investigation was conducted in preparation for the upcoming first-in-human study. We investigated 3 wound dressings containing silver (Acticoat $^{\mathrm{TM}}$, Mepilex $^{\circledR} \mathrm{Ag}$, and $\mathrm{Urgotul}^{\circledR}$ SSD) and 2 without silver (Suprathel ${ }^{\circledR}$ and Vaseline Gauze ${ }^{\mathrm{TM}}$ ). These dressings had already been investigated in preclinical studies regarding their adverse effects on cells, ${ }^{21,22,40,41}$ bacteria, ${ }^{42-44}$ created wounds,${ }^{45,46}$ and in clinical applications. ${ }^{47-53}$

At day 10 we observed a graft take of $100 \%$. After 21 days, the same macroscopic results were found, and histologically, every specimen demonstrated a stratified and cornified epidermis. This was supported by the presence of epidermal differentiation markers, such as CKl, involucrin, and loricrin. Host blood vessels were detected in the human dermal part of all the transplanted skin substitutes. In samples from the Mepilex ${ }^{\circledR}$ AG-group, blood vessels were detected in the graft, but not as close to the surface as was seen with the other groups. Based on experience, this might be due to inter-animal variations, rather than the wound dressing used. Rat immune cells, such as granulocytes, could be detected only rarely, highlighting that the human skin substitutes are well-accepted in this model. To summarize, a well-vascularized human dermis was established by the host vasculature without any signs of immune reaction in all transplants using silver and non-silvercontaining wound dressings. This was also observed in our previous studies when only a silicon foil dressing was applied. ${ }^{35,54}$

Our dressing schedule deserves a more detailed comment. After 10 days we changed the wound dressing in every specimen to silicone foil, since there is no beneficial effect of silver dressings with respect to infection or wound healing after this time period, as per the current literature. ${ }^{5,56}$

Importantly, none of the tested wound dressings reduced the survival or number of human epithelial cells within the skin substitutes. This is particularly notable for the silver-containing dressings, which have demonstrated a cytotoxic effect in previous studies. The study by Esteban-Vives et $\mathrm{al}^{57}$ described a negative effect of Mepilex $A G^{\circledR}$ on keratinocyte attachment and survival in vitro. Moreover, in the current study, the formation of a multilayered stratified epidermis was not hampered, either with or without silver, which has also been described previously. ${ }^{21,22,40,41}$

Also of note, we observed an imprint of the mesh structure of Vaseline Gauze $^{\mathrm{TM}}$, Urgotul ${ }^{\circledR}$ SSD, and Acticoat ${ }^{\mathrm{TM}}$ onto the skin substitutes. This was a transient finding and did not affect the epidermal structure or biological viability.

We did not investigate the presence of released, absorbed, or distributed silver in the host organism during the study. Related investigations have previously been performed by others. ${ }^{21,22,46}$ Nonetheless, we also have to take into account that we used an excisional wound model on athymic nude rats to test our human dermo-epidermal skin substitutes. Valvis et $\mathrm{al}^{58}$ in a mouse model (using C57BL/6 mice) showed significant differences between immune responses in mice comparing burn and excisional injuries. They observed an acute neutrophil attractant with a delayed macrophage promoting environment in the excisional injury, compared to an early macrophage promoting environment after a burn injury.

However, we used athymic rats and applied human cellularized skin substitutes onto excisional created wounds also affecting the wound healing response, and tested for macrophages and granulocytes only at a later time point but not at the acute wound healing phase. Of course, our preclinical animal experiments give hints about possible processes taking place in a skin wound healing situation but are not completely comparable to a clinical human situation.

In conclusion, all herein tested dressings applied on our clinically GMP grade human dermo-epidermal skin substitutes yielded a satisfying outcome regarding graft take, epithelialization, and infection control. Therefore, all tested wound dressings 
would be suitable for use in the foreseen phase I clinical trial. Furthermore, our large autologous skin substitutes demonstrated stability in combination with every tested dressing. Concerns about the cytotoxicity of silver were not validated during the study. In fact, we observed reproducible results with excellent graft take with the silver-containing dressings. If there is widespread use of our skin substitutes in the future, this study supports the use of silver-containing dressings, particularly when infection control is necessary.

\section{ACKNOWLEDGMENTS}

We are particularly grateful to the Fondation Gaydoul and the sponsors of "Dona Tissue" (Thérèse Meier and Robert Zingg) for their financial support and interest in our work. The funding sources did not participate in the study design, the collection, analysis and interpretation of data, the writing of the report, or the decision to submit the article for publication.

\section{REFERENCES}

1. Rheinwald JG, Green H. Epidermal growth factor and the multiplication of cultured human epidermal keratinocytes. Nature 1977;265:421-4.

2. Rheinwald JG, Green H. Serial cultivation of strains of human epidermal keratinocytes: the formation of keratinizing colonies from single cells. Cell 1975;6:331-43.

3. Gallico GG 3rd, O'Connor NE, Compton CC, Kehinde $\mathrm{O}$, Green H. Permanent coverage of large burn wounds with autologous cultured human epithelium. N Engl J Med 1984;311:448-51.

4. Boyce ST, Goretsky MJ, Greenhalgh DG, Kagan RJ, Rieman MT, Warden GD. Comparative assessment of cultured skin substitutes and native skin autograft for treatment of fullthickness burns. Ann Surg 1995;222:743-52.

5. Boyce ST, Kagan RJ, Greenhalgh DG, et al. Cultured skin substitutes reduce requirements for harvesting of skin autograft for closure of excised, full-thickness burns. J Trauma 2006;60:821-9.

6. Boyce ST, Simpson PS, Rieman MT, et al. Randomized, paired-site comparison of autologous engineered skin substitutes and split-thickness skin graft for closure of extensive, full-thickness burns. J Burn Care Res 2017. doi:10.1097/ BCR.0000000000000401.

7. Shakespeare PG. The role of skin substitutes in the treatment of burn injuries. Clin Dermatol 2017;23:413-8.

8. Edmonds M. Apligraf in the treatment of neuropathic diabetic foot ulcers. Int J Low Extrem Wounds 2009;8:11-8.

9. Barber C, Watt A, Pham C, et al. Influence of bioengineered skin substitutes on diabetic foot ulcer and venous leg ulcer outcomes. J Wound Care 2008;17:517-27.

10. Waymack P, Duff RG, Sabolinski M. The effect of a tissue engineered bilayered living skin analog, over meshed splitthickness autografts on the healing of excised burn wounds. The Apligraf Burn Study Group. Burns 2000;26:609-19.

11. Pontiggia L, Biedermann T, Meuli M, et al. Markers to evaluate the quality and self-renewing potential of engineered human skin substitutes in vitro and after transplantation. J Invest Dermatol 2009;129:480-90.

12. Braziulis E, Diezi M, Biedermann T, et al. Modified plastic compression of collagen hydrogels provides an ideal matrix for clinically applicable skin substitutes. Tissue Eng Part C Methods 2012;18:464-74.

13. Hartmann-Fritsch F, Biedermann T, Braziulis E, Meuli M, Reichmann E. A new model for preclinical testing of dermal substitutes for human skin reconstruction. Pediatr Surg Int 2013;29:479-88.

14. Biedermann T, Klar AS, Böttcher-Haberzeth S, Schiestl C, Reichmann E, Meuli M. Tissue-engineered dermo-epidermal skin analogs exhibit de novo formation of a near natural neurovascular link 10 weeks after transplantation. Pediatr Surg Int 2014;30:165-72.

15. Böttcher-Haberzeth S, Biedermann T, Klar AS, et al. Characterization of pigmented dermo-epidermal skin substitutes in a long-term in vivo assay. Exp Dermatol 2015;24:16-21.

16. Böttcher-Haberzeth S, Biedermann T, Reichmann E. Tissue engineering of skin. Burns 2010;36:450-60.

17. Lumenta DB, Kamolz LP, Frey M. Adult burn patients with more than $60 \%$ TBSA involved-Meek and other techniques to overcome restricted skin harvest availability - the Viennese Concept. J Burn Care Res 2009;30:231-42.

18. Rennekampff HO, Rabbels J, Reinhard V, Becker ST, Schaller HE. Comparing the Vancouver Scar Scale with the cutometer in the assessment of donor site wounds treated with various dressings in a randomized trial. J Burn Care Res 2006;27:345-51.

19. Atiyeh BS, Costagliola M. Cultured epithelial autograft (CEA) in burn treatment: three decades later. Burns 2007;33:405-13.

20. Barret JP, Herndon DN. Effects of burn wound excision on bacterial colonization and invasion. Plast Reconstr Surg 2003;111:744-50; discussion 751-2.

21. Burd A, Kwok CH, Hung SC, et al. A comparative study of the cytotoxicity of silver-based dressings in monolayer cell, tissue explant, and animal models. Wound Repair Regen 2007;15:94-104.

22. Duc Q1, Breetveld M, Middelkoop E, Scheper RJ, Ulrich MM, Gibbs S. A cytotoxic analysis of antiseptic medication on skin substitutes and autograft. Br J Dermatol 2007; 157:33-40.

23. Fong J, Wood F. Nanocrystalline silver dressings in wound management: a review. Int J Nanomedicine 2006;1:441-9.

24. Hamnett N, Chandra A, Chamaidi E, Farroha A. Improving and maintaining conformity of Acticoat ${ }^{\circledR}$ dressings with shear reducing transfixion suture. J Burn Care Res 2016;37:e398-9

25. Hassan S, Reynolds G, Clarkson J, Brooks P. Challenging the dogma: relationship between time to healing and formation of hypertrophic scars after burn injuries. J Burn Care Res 2014;35:el18-24.

26. Sheckter CC, Van Vliet MM, Krishnan NM, Garner WL. Cost-effectiveness comparison between topical silver sulfadiazine and enclosed silver dressing for partial-thickness burn treatment. J Burn Care Res 2014;35:284-90.

27. Atiyeh BS, Hayek SN. The safety and efficacy of dressings with silver - addressing clinical concerns. Int Wound J 2007;4:283-4.

28. Aziz Z, Abu SF, Chong NJ. A systematic review of silvercontaining dressings and topical silver agents (used with dressings) for burn wounds. Burns 2012;38:307-18.

29. Leaper DJ. Silver dressings: their role in wound management. Int Wound J 2006;3:282-94.

30. Marx DE, Barillo DJ. Silver in medicine: the basic science. Burns 2014;40 (Suppl 1):S9-S18.

31. Walker M, Parsons D. The biological fate of silver ions following the use of silver-containing wound care products - a review. Int Wound J 2014;11:496-504.

32. Hartmann-Fritsch F, Marino D, Reichmann E. About ATMPs, SOPs and GMP : the hurdles to produce novel skin grafts for clinical use. Transfus Med Hemotherapy. 2016;43:344-352. 
33. Biedermann T, Pontiggia L, Böttcher-Haberzeth S, et al. Human eccrine sweat gland cells can reconstitute a stratified epidermis. J Invest Dermatol 2010;130:1996-2009.

34. Böttcher-Haberzeth S, Biedermann T, Schiestl C, et al. Matriderm ${ }^{\circledR} 1 \mathrm{~mm}$ versus Integra ${ }^{\circledR}$ Single Layer $1.3 \mathrm{~mm}$ for one-step closure of full thickness skin defects: a comparative experimental study in rats. Pediatr Surg Int 2012;28:171-7.

35. Klar AS, Böttcher-Haberzeth S, Biedermann T, Schiestl C, Reichmann E, Meuli M. Analysis of blood and lymph vascularization patterns in tissue-engineered human dermo-epidermal skin analogs of different pigmentation. Pediatr Surg Int 2014;30:223-31.

36. Lichti U, Anders J, Yuspa SH. Isolation and short-term culture of primary keratinocytes, hair follicle populations and dermal cells from newborn mice and keratinocytes from adult mice for in vitro analysis and for grafting to immunodeficient mice. Nat Protoc 2008;3:799-810.

37. Supp AP, Neely AN, Supp DM, Warden GD, Boyce ST. Evaluation of cytotoxicity and antimicrobial activity of Acticoat burn dressing for management of microbial contamination in cultured skin substitutes grafted to athymic mice. J Burn Care Rehabil 2005;26:238-46.

38. Braziulis E, Biedermann T, Hartmann-Fritsch F, et al. Skingineering I: engineering porcine dermo-epidermal skin analogues for autologous transplantation in a large animal model. Pediatr Surg Int 2011;27:241-7.

39. Schiestl C, Biedermann T, Braziulis E, et al. Skingineering II: transplantation of large-scale laboratory-grown skin analogues in a new pig model. Pediatr Surg Int 2011;27:249-54.

40. Matoušková E, Mestak O. The effect of different biological and biosynthetic wound covers on keratinocyte growth, stratification and differentiation in vitro. J Tissue Eng 2014;5:2041731414554966.

41. Boonkaew B, Barber PM, Rengpipat S, et al. Development and characterization of a novel, antimicrobial, sterile hydrogel dressing for burn wounds: single-step production with gamma irradiation creates silver nanoparticles and radical polymerization. J Pharm Sci 2014;103:3244-53.

42. Cavanagh MH, Burrell RE, Nadworny PL. Evaluating antimicrobial efficacy of new commercially available silver dressings. Int Wound J 2010;7:394-405.

43. Halstead FD, Rauf M, Bamford A, et al. Antimicrobial dressings: comparison of the ability of a panel of dressings to prevent biofilm formation by key burn wound pathogens. Burns 2015;41:1683-94.

44. Ryssel H, Germann G, Riedel K, Reichenberger M, Hellmich $S$, Kloeters O. Suprathel-acetic acid matrix versus acticoat and aquacel as an antiseptic dressing: an in vitro study. Ann Plast Surg 2010;65:391-5.
45. Hiro ME, Pierpont YN, Ko F, Wright TE, Robson MC, Payne WG. Comparative evaluation of silver-containing antimicrobial dressings on in vitro and in vivo processes of wound healing. Eplasty 2012;12:e48.

46. Pfurtscheller K, Petnehazy T, Goessler W, Bubalo V, Kamolz LP, Trop M. Transdermal uptake and organ distribution of silver from two different wound dressings in rats after a burn trauma. Wound Repair Regen 2014;22:654-9.

47. Jester I, Bohn I, Hannmann T, Waag KL, Loff S. Comparison of two silver dressings for wound management in pediatric burns. Wounds 2008;20:303-8.

48. Glat PM, Zhang SH, Burkey BA, Davis WJ. Clinical evaluation of a silverimpregnated foam dressing in paediatric partial-thickness burns. J Wound Care. 2015;24:s4-s10.

49. Everett M, Massand S, Davis W, Burkey B, Glat PM. Use of a copolymer dressing on superficial and partial-thickness burns in a paediatric population. J Wound Care. 2015;24:S4-S8.

50. Highton L, Wallace C, Shah M. Use of Suprathel® for partial thickness burns in children. Burns 2013;39:136-41.

51. Moiemen NS, Shale E, Drysdale KJ, Smith G, Wilson YT, Papini R. Acticoat dressings and major burns: systemic silver absorption. Burns 2011;37:27-35.

52. Tang H, Lv G, Fu J, et al. An open, parallel, randomized, comparative, multicenter investigation evaluating the efficacy and tolerability of Mepilex Ag versus silver sulfadiazine in the treatment of deep partial-thickness burn injuries. J Trauma Acute Care Surg 2015;78:1000-7.

53. Gee Kee E, Kimble RM, Cuttle L, Stockton K. Comparison of three different dressings for partial thickness burns in children: study protocol for a randomised controlled trial. Trials $2013 ; 14: 403$.

54. Hartmann-Fritsch F, Biedermann T, Braziulis E, et al. Collagen hydrogels strengthened by biodegradable meshes are a basis for dermo-epidermal skin grafts intended to reconstitute human skin in a one-step surgical intervention. J Tissue Eng Regen Med 2016;10:81-91.

55. Leaper D. Appropriate use of silver dressings in wounds: international consensus document. Int Wound J 2012;9:461-4.

56. Brown M, Dalziel SR, Herd E, Johnson K, Wong She R, Shepherd M. A randomized controlled study of silver-based burns dressing in a pediatric emergency department. J Burn Care Res 2016;37:e340-7.

57. Esteban-Vives R, Young MT, Ziembicki J, Corcos A, Gerlach JC. Effects of wound dressings on cultured primary keratinocytes. Burns 2016;42:81-90.

58. Valvis SM, Waithman J, Wood FM, Fear MW, Fear VS. The immune response to skin trauma is dependent on the etiology of injury in a mouse model of burn and excision. J Invest Dermatol 2015;135:2119-28. 